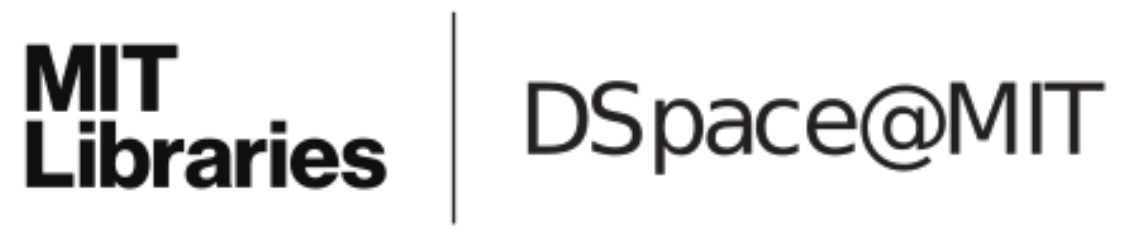

\author{
MIT Open Access Articles
}

Electro-osmotic flow through a two-dimensional screen-pump filter

The MIT Faculty has made this article openly available. Please share how this access benefits you. Your story matters.

Citation: Liu, Ying-Hong et al. "Electro-osmotic flow through a two-dimensional screen-pump filter." Physical Review E 84.3 (2011): n. pag. Web. 25 Jan. 2012. (C) 2011 American Physical Society

As Published: http://dx.doi.org/10.1103/PhysRevE.84.036301

Publisher: American Physical Society (APS)

Persistent URL: http://hdl.handle.net/1721.1/68653

Version: Final published version: final published article, as it appeared in a journal, conference proceedings, or other formally published context

Terms of Use: Article is made available in accordance with the publisher's policy and may be subject to US copyright law. Please refer to the publisher's site for terms of use. 
PHYSICAL REVIEW E 84, 036301 (2011)

\title{
Electro-osmotic flow through a two-dimensional screen-pump filter
}

\author{
Ying-Hong Liu* \\ Institute of Applied Mechanics, National Taiwan University, Taipei 106, Taiwan, ROC
}

Chih-Yu Kuo ${ }^{\dagger}$

Division of Mechanics, Research Center for Applied Sciences, Academia Sinica, Taipei 115, Taiwan, ROC

Chien C. Chang ${ }^{\ddagger}$

Institute of Applied Mechanics, National Taiwan University, Taipei 106, Taiwan, ROC

Chang-Yi Wang $\$$

Departments of Mathematics and Mechanical Engineering, Michigan State University, East Lansing, Michigan 48824, USA

(Received 16 March 2011; revised manuscript received 20 July 2011; published 1 September 2011)

\begin{abstract}
The electro-osmotic flow driven by a screen pump, composed of a line array of evenly spaced identical rectangular solid blocks, is investigated under the Debye-Hückel approximation. The geometry of the screen pump is determined by the spacing and aspect ratio of the solid blocks. A constant surface zeta potential is assumed on the block surface. The method of eigenfunction series expansion is applied to solve analytically for the applied electric field, electric charge potential in the fluid, and flow field. Because of the low Reynolds number, Stokes equations are applied for the flow. The analytic result is first confirmed by comparing with the exact solution of the electro-osmotic flow in an infinite channel. Then different geometries of the screen pump and the effect of the electrokinetic width are computed for their influence on the flow rate. Recirculating eddies and reversing flow are found even though the applied electric driving field is unidirectional.
\end{abstract}

DOI: 10.1103/PhysRevE.84.036301

PACS number(s): 47.52.+j, 47.65.-d, 47.63.mf, 47.15.G-

\section{INTRODUCTION}

Recently, microfluidic devices have been playing important roles in scientific and engineering research due to their wide applications in microfluid transport and mixing, microbiological sensors, and biomedical instruments [1-10]. One of the important fluid-driving mechanisms in these laboratory-ona-chip (LOC) applications is the electro-osmotic (EO) flow. It is the result of the interaction between the electric double layer (EDL) and an applied external electric potential field. The advantage of this kind of device is that one does not need to apply large pressure to overcome the viscous resistance for driving fluid in the microchannels. The fabrication of the EO devices is in general simpler because no moving parts are required [11].

The EO pumping mechanism is the result of the interaction between the applied external electric field and the electric charge potential in the fluid. The electric charge is caused by the redistribution of ion concentration in the fluid due to the surface zeta potential on interfaces against the fluid. This electrical charged layer is referred to as the EDL. Once applied with electric field, the electrokinetic body force is formed and drives the fluid. In common applications, the applied electric

\footnotetext{
*Department of Civil Engineering, Massachusetts Institute of Technology, Cambridge, MA 02139, USA; yinghung@mit.edu

†cykuo06@gate.sinica.edu.tw

${ }^{\ddagger}$ Division of Mechanics, Research Center for Applied Sciences, Academia Sinica, Taipei 115, Taiwan, ROC.; Taida Institute of Mathematical Sciences, National Taiwan University, Taipei 106, Taiwan, ROC; mechang@webmail.iam.ntu.edu.tw

§cywang@math.msu.edu
}

field is a unidirectional static field, and, subsequently, the EO force results in a shear layer flow. In the shear layer, the flow speed increases exponentially and asymptotes to the free-flow field outside the EDL as increasing the distance to the interface. This sharp velocity profile may enhance the mixing or dispersion of sediments in the shear layer [12]. This property leads to the flow in a straight fluidic channel forming a pluglike velocity profile rather than a parabolic (Poseuille) shape as in pressure-driven flows. In contrast, this may reduce some desired flow motion in the free flow, such as mixing.

Therefore, more sophisticated proposals have been emerging for special circumstances, such as the AC electro-osmotic pump for high flow rates [1] or for controlling the flow direction [13]. Nowadays, multicomponents are often integrated on LOC devices to perform multistage laboratory functions. These components include mixers, screen filters, flow splitters, etc. The interaction of these components with the external electric field is one of the major concerns in EO devices. In the present paper, we consider a screen filter, which is composed of a line array of evenly spaced identical rectangular blocks (Fig. 1). Because of their surface zeta potential, when applied with an external electric field, the device component itself generates electrokinetic pumping forces. Hence, we also refer to this screen filter as a screen pump. In laboratories, a similar screen filter is applied to capture suspended particles, such as cells and vescules in LOC devices [14,15].

To focus solely on the electrokinetic mechanism, we investigate the EO flow without applying an external driving pressure across the fluidic channel. In Sec. II we illustrate the basic theory and the governing equations. The method of eigenfunction series expansion is applied to solve for the applied electric potential field, the EDL potential field, as 
(a)

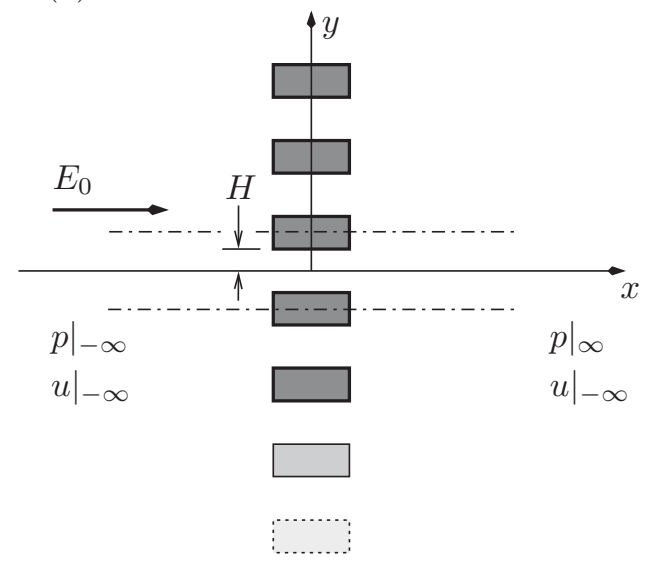

(b)

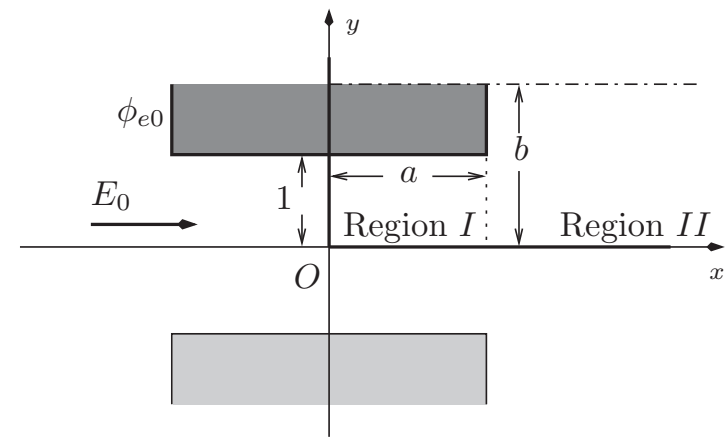

FIG. 1. (a) The cross section of the two-dimensional screen pump where the electric-osmotic flow passes through. The half of the channel width is $H$, which is used to normalize lengths. The portion between the dash-dotted lines is the unit section of the screen pump. (b) The unit section of the pump. The lengths have been normalized, given the (half) channel length $a$ and the (half) width of the free-flow field $b$. Due to symmetry, we need only to solve for the domain in the first quadrant. The domain is decomposed into two regions such that Region I is inside the channel between the neighboring blocks and Region II is the semi-infinite free-flow strip.

well as the flow field. The solution of the flow around the screen pump is introduced [16] to obtain the analytic flow field for the entire domain of interest. In Sec. III we verify the the analytic series solution with the exact solution for an infinite microchannel, a degenerated special case of the present screen pump. Then the geometry of the filter is varied, and its relation with the flux and flow field is computed and the effect of the electrokinetic width is investigated. It is observed that recirculating eddies can form under some particular configurations, and they can even lead to reversing flows even when the external electric field is unidirectional.

\section{BASIC THEORY}

The governing equation for a steady electro-osmotic (EO) flow, driven by an applied electric potential field $\phi_{p}^{*}$, at a low Reynolds number is [11]

$$
-\vec{\nabla}^{*} p^{*}+\mu \nabla^{* 2} \vec{u}^{*}+\rho_{e} \vec{\nabla}^{*} \phi_{p}^{*}=0,
$$

where $p^{*}$ is the pressure, $\mu$ is the fluid viscosity, and $\vec{u}^{*}$ is the velocity, respectively. The third term on the left-hand side is the electrokinetic force, which results from the interaction between the applied electric field and the charge density $\rho_{e}$ in the fluid. The electric charge density in a simple two-ion electrolic fluid is described by the nonlinear PossionBoltzmann equation

$$
\rho_{e}=-\epsilon \nabla^{* 2} \phi_{e}^{*}=-2 z e n_{0} \sinh \left(\frac{z e \phi_{e}^{*}}{k_{B} T}\right),
$$

where $\epsilon$ is the dielectric constant, $\phi_{e}^{*}$ is the EDL potential, $e$ is the electron charge, $n_{0}$ is the bulk electrolyte concentration of a binary electrolyte dissociating into cations and anions of valence $z, k_{B}$ is the Boltzmann constant, and $T$ is the temperature. The EDL forming at the flow boundaries provides the shield for the electric potential such that the applied electric potential can be described by

$$
\nabla^{2} \phi_{p}^{*}=0
$$

with vanishing normal derivatives of the potential on the solid boundaries.

Next, let us normalize the governing equations. If the charge potential is a small quantity compared to the thermal energy of the ions, i.e., the ratio $z e \phi_{e}^{*} / k_{B} T$ is much less than one, Eq. (2) can be approximated by

$$
\rho_{e}=-\epsilon \nabla^{* 2} \phi_{e}^{*}=-2 z e n_{0}\left(\frac{z e \phi_{e}^{*}}{k_{B} T}\right),
$$

which is referred to as the Debye-Hückel equation. Introducing $\phi_{e} \rightarrow \phi_{e}^{*} / \phi_{e 0}$, with the constant zeta potential on the block boundaries $\phi_{e 0}$, and choosing the half width of the channel $H$ as the characteristic length for all of the length scales, as shown in Fig. 1, we normalize Eq. (4) into

$$
\nabla^{2} \phi_{e}=K^{2} \phi_{e}
$$

where $K^{2}$ is defined as $2 z^{2} e^{2} n_{0} H^{2} / \epsilon k_{B} T . K$ is called the Debye-Hückel parameter and is related to the inverse of the electrokinetic width, or the thickness of the EDL.

Further normalizing each physical parameters by the substitutions $p \rightarrow \epsilon E_{0} \phi_{e 0}^{*} p^{*} / H, \vec{u} \rightarrow \epsilon E_{0} \phi_{e 0} \vec{u}^{*} / \mu, \phi_{p} \rightarrow$ $\phi_{p}^{*} /\left(E_{0} H\right)$ with $E_{0}$ being the characteristic value of the applied external electric field, we obtain the nondimensionalized equation

$$
-\vec{\nabla} p+\nabla^{2} \vec{u}-K^{2} \phi_{e} \vec{\nabla} \phi_{p}=0
$$

for the fluid and

$$
\nabla^{2} \phi_{p}=0
$$

for the applied electric field. The fluid pressure in Eq. (6) can be eliminated by introducing a stream function $\psi$, such that $\vec{u}=(\partial \psi / \partial y,-\partial \psi / \partial x)$, and by taking the curl $(\vec{\nabla} \times)$ operation on the equation. This yields the resulting governing equation for the electro-osmotic flow as a biharmonic equation of the stream function:

$$
\nabla^{4} \psi=K^{2}\left(\frac{\partial \phi_{e}}{\partial y} \frac{\partial \phi_{p}}{\partial x}-\frac{\partial \phi_{e}}{\partial x} \frac{\partial \phi_{p}}{\partial y}\right) .
$$


Equations (5), (7), and (8) are the equation system for the EO flow around the screen pump. Because the equations are linear and because of the symmetry of the screen pump [Fig. 1(a)], the system is solved in a unit section of the pump, as shown in Fig. 1(b). The unit section is divided into two regions: Regions I and II. Continuity conditions are applied on the joint plane between the two regions to determine the eigenfunction expansion series. The procedures are discussed in detail in the following subsections.

\section{A. Applied electric potential field}

The flow is purely driven by the applied electric field. As $x \rightarrow \pm \infty$, the applied electric field is free from the influence of the pump, and, therefore, the associated electric potential is a linear function. Normalizing the electric potential by the field strength $E_{0}$, we can express the series solution of the potential [Eq. (7)] in Region II as

$$
\phi_{p \mathrm{II}}(x, y)=-x+B_{0}^{p}+\sum_{n=1}^{\infty} B_{n}^{p} e^{-\beta_{n}^{p}(x-a)} \cos \left(\beta_{n}^{p} y\right),
$$

where $\beta_{n}^{p}=n \pi / b$ and the solution in Region I as

$$
\phi_{p \mathrm{I}}(x, y)=-A_{0}^{p} x+\sum_{n=1}^{\infty} A_{n}^{p} \sinh \left(\alpha_{n}^{p} x\right) \cos \left(\alpha_{n}^{p} y\right),
$$

where $\alpha_{n}^{p}=n \pi$. Note that the solutions are even, i.e., periodic in the $y$ direction.

The coefficients $A_{n}^{p}, B_{n}^{p}$ are the unknowns that have to be determined by applying the continuity conditions of the electric potential at $x=a$ :

$$
\begin{aligned}
\phi_{p \mathrm{I}}(a, y) & =\phi_{p \mathrm{II}}(a, y), \quad 0 \leqslant y \leqslant 1, \\
\frac{\partial \phi_{p \mathrm{I} x}}{\partial x}(a, y) & =\frac{\partial \phi_{p \mathrm{II}}}{\partial x}(a, y), \quad 0 \leqslant y<1 .
\end{aligned}
$$

On the vertical downstream-facing surface $(x=a, 1 \leqslant y \leqslant b)$ of the pump, the normal derivative of the electric potential vanishes:

$$
\frac{\partial \phi_{p \mathrm{II}}}{\partial x}(a, y)=0, \quad 1 \leqslant y \leqslant b .
$$

For numerical calculation, we need to truncate the infinite solution series [Eqs. (9) and (10)] into a finite size. This is done by discretizing the joining line $(x=a, 0 \leqslant y \leqslant b)$ into a set of equally spaced points $\left(a, y_{n}\right)$. Applying the continuity and boundary conditions [Eqs. (11) and (12)], we form a linear algebraic system for the unknown coefficients. Figure 2 sketches the (normalized) electric potential when an electric field is applied to a screen filter of $a=1.0$ and $b=1.5$. The electric field $\left(-\vec{\nabla} \phi_{p}\right)$ is mostly uniform and toward the positive $x$ direction except in the region near the joining corner of the fluid channel. In addition, approaching to the screen block surface, the electric field turns parallel to the block surface.

\section{B. EDL potential field}

Under the Debye-Hückel approximation, the EDL potential degenerates to the modified Helmholtz equation [Eq. (5)]. The EDL potential on the solid boundary normalized by

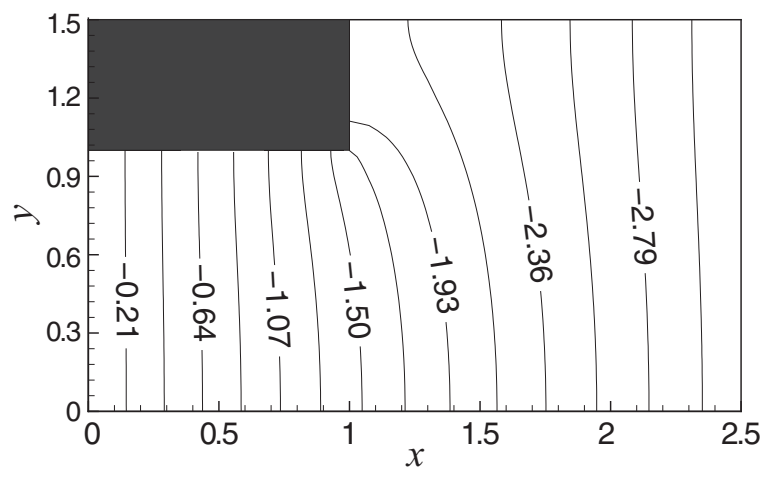

FIG. 2. The potential $\phi_{p}$ of the applied electric field. The screen filter geometry is $a=1.0, b=1.5$.

the surface zeta potential is one. Therefore, in Region I, the EDL potential is easily solved by letting $\phi_{e \mathrm{I}}=1-\chi=$ $1-\sum_{n=1}^{\infty} f_{n}(x) \cos \left(\beta_{n}^{e} y\right)$, where $\chi$ satisfies

$$
\nabla^{2} \chi-K^{2} \chi=-K^{2}
$$

with the boundary condition $\chi=0$ on the solid surface. Substituting the identity $1=\sum_{n=1}^{\infty} a_{n} \cos \left(\beta_{n}^{e} y\right)$ into Eq. (13) and after some algebra, we can express the EDL potential in terms of the series expansion

$$
\phi_{e \mathrm{I}}(x, y)=1-\sum_{n=1}^{\infty}\left[\frac{K^{2} a_{n}}{\left(\beta_{n}^{e}\right)^{2}+K^{2}}+A_{n}^{e} \cosh \left(\alpha_{n}^{e} x\right)\right] \cos \left(\beta_{n}^{e} y\right),
$$

and

$$
\phi_{e \mathrm{II}}(x, y)=B_{0}^{e} e^{-K(x-a)}+\sum_{n=1}^{\infty} B_{n}^{e} e^{-\delta_{n}^{e}(x-a)} \cos \left(\gamma_{n}^{e} y\right),
$$

where $\quad \beta_{n}^{e}=\left(n-\frac{1}{2}\right) \pi, \quad a_{n}=2(-1)^{n+1} / \beta_{n}^{e}, \quad \alpha_{n}^{e}=$ $\sqrt{\left(\beta_{n}^{e}\right)^{2}+K^{2}}, \quad \gamma_{n}^{e}=n \pi / b, \quad$ and $\delta_{n}^{e}=\sqrt{\left(\gamma_{n}^{e}\right)^{2}+K^{2}}$. The Region I solution satisfies the constant value 1 on $0 \leqslant x \leqslant a$, $y=1$, automatically. The Region II solution, in addition, decays exponentially in the $x$ direction away from the filter. From the symmetry, the EDL potential is symmetric with respect to $x=0$ and periodic in the $y$ direction.

Similar to the electric potential field, the unknown coefficients $A_{n}^{e}$ and $B_{n}^{e}$ are to be determined by the continuity conditions at $x=a, 0 \leqslant y \leqslant 1$ :

$$
\begin{gathered}
\phi_{e \mathrm{I}}(a, y)=\phi_{e \mathrm{II}}(a, y), \quad 0 \leqslant y<1, \\
\frac{\partial \phi_{e \mathrm{I}}}{\partial x}(a, y)=\frac{\partial \phi_{e \mathrm{II}}}{\partial x}(a, y), \quad 0 \leqslant y \leqslant 1,
\end{gathered}
$$

and the zeta potential on the vertical downstream-facing surface

$$
\phi_{e \mathrm{II}}(a, y)=1, \quad 1 \leqslant y \leqslant b .
$$

A numerical scheme similar to the one described in Sec. II A is applied to solve for the EDL potential. The only difference is that the solution series and the continuity and boundary conditions are replaced by Eqs. (14), (15), (16), and (17). 


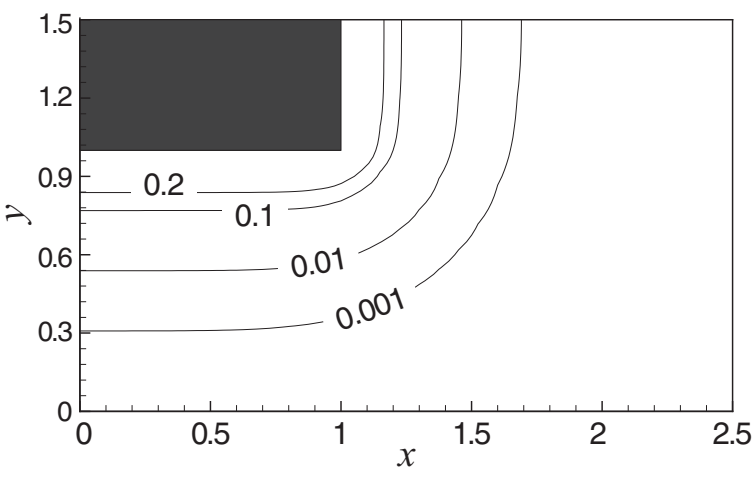

FIG. 3. The EDL potential, $\phi_{e}$. The screen filter geometry is $a=$ $1.0, b=1.5$, and the Debye-Hückel parameter is $K=10$.

Figure 3 sketches the EDL potential for Debye-Hückel parameter $K=10$. The geometry of the screen filter is $a=1.0$ and $b=1.5$. For this moderate $K$, we clearly identify that the EDL symmetrically surrounds the screen filter block at a thickness about 0.5 (from the surface to $\phi_{e}=0.01$ ). As the Debye-Hückel parameter increases, the EDL thickness decreases.

\section{Flow field solution}

The flow field is described by the stream function, which satisfies Eq. (8). This equation can be solved systematically by decomposing the stream function into the sum of a particular solution and a homogeneous solution, i.e., $\psi=\psi_{p}+\psi_{h}$. The particular solution $\psi_{p}$ balances the electrokinetic force, and the homogeneous solution $\psi_{h}$ is chosen to make the resultant solution fulfill the boundary conditions.

The particular solutions for both Regions I and II, though tedious, can be found straightforwardly by substituting the potentials, Eqs. (9), (10) (14), and (15) into the right-hand side of Eq. (8) and, subsequently, integrating the resulting series. The particular solution $\psi_{p \mathrm{I}}(x, y)$ of Region $\mathrm{I}$ is shown in the Appendix.

For simplicity, we have chosen the particular solution in Region I by setting the integration constant zero such that the solution is symmetric with respect to $x=0$ and satisfies the constant datum, $\psi_{\mathrm{I} p}(x, 0)=0$, i.e., no flow cross the center line of the channel, and the no-slip condition, $\left(\partial \psi_{\mathrm{I} p} / \partial y\right)(x, 1)=0$, on the block surface.

Accordingly, we obtain the particular solution for Region II:

$$
\begin{aligned}
\psi_{p \mathrm{II}}(x, y)= & \sum_{n=1}^{\infty} P_{n}^{1} e^{-\delta_{n}^{e}(x-a)} \sin \left(\gamma_{n}^{e} y\right) \\
& +\sum_{n=1}^{\infty} P_{n}^{2} e^{-\left(K+\beta_{n}^{p}\right)(x-a)} \sin \left(\beta_{n}^{p} y\right) \\
& +\sum_{n, m=1}^{\infty} P_{m n}^{3} e^{-\left(\delta_{n}^{e}+\beta_{m}^{p}\right)(x-a)} \sin \left[\left(\delta_{n}^{e}-\beta_{m}^{p}\right) y\right] \\
& +\sum_{n, m=1}^{\infty} P_{m n}^{4} e^{-\left(\delta_{n}^{e}+\beta_{m}^{p}\right)(x-a)} \sin \left[\left(\delta_{n}^{e}+\beta_{m}^{p}\right) y\right]
\end{aligned}
$$

where

$$
\begin{gathered}
P_{n}^{1}=K^{2} \frac{B_{n}^{e} \gamma_{n}^{e}}{\left[\left(\delta_{n}^{e}\right)^{2}-\left(\gamma_{n}^{e}\right)^{2}\right]^{2}}, \\
P_{n}^{2}=-K^{3} \frac{B_{0}^{e} B_{n}^{p} \beta_{n}^{p}}{\left[\left(K+\beta_{n}^{p}\right)^{2}-\left(\beta_{n}^{p}\right)^{2}\right]^{2}}, \\
P_{m n}^{3}=K^{2} \frac{B_{n}^{e} B_{m}^{p} \gamma_{n}^{e} \beta_{m}^{p}+B_{n}^{e} B_{m}^{p} \delta_{n}^{e} \beta_{m}^{p}}{2\left[\left(\delta_{n}^{e}+\beta_{m}^{p}\right)^{2}-\left(\gamma_{n}^{e}-\beta_{m}^{p}\right)^{2}\right]^{2}}, \\
P_{m n}^{4}=K^{2} \frac{B_{n}^{e} B_{m}^{p} \gamma_{n}^{e} \beta_{m}^{p}-B_{n}^{e} B_{m}^{p} \delta_{n}^{e} \beta_{m}^{p}}{2\left[\left(\delta_{n}^{e}+\beta_{m}^{p}\right)^{2}-\left(\gamma_{n}^{e}+\beta_{m}^{p}\right)^{2}\right]^{2}} .
\end{gathered}
$$

The particular solution is chosen to satisfy the constant datum on the channel center line. The solution automatically vanishes on $y=b$.

The continuity and boundary conditions are to be satisfied by the resulting stream function. This is done by adjusting the homogeneous solution to Eq. (8), combined with the particular solutions in the two regions [Eqs. (A1) and (18)]. The boundary conditions of the flow are given in the following paragraph.

Because the geometric configuration is periodic in the $y$ direction, there is no flow crossing the solid boundaries depicted in Fig. 1. On these boundaries, the stream function is constant. We set the stream function to the datum value on the center line of the channel:

$$
\psi_{\mathrm{I}}(x, 0)=\psi_{\mathrm{II}}(x, 0)=0,
$$

and an unknown constant $q$ on the block surface and at $y=b$ in Region II:

$$
\left\{\begin{array}{l}
\psi_{\mathrm{I}}(x, 1)=\psi_{\mathrm{II}}(x, b)=q, \\
\psi_{\mathrm{II}}(a, y)=q, \quad 1 \leqslant y \leqslant b .
\end{array}\right.
$$

From the definition of the stream function, we can easily seen that $q$ is the volume flow rate of the unit section. In addition, the vorticity, $-\nabla^{2} \psi$, is zero on the fluid symmetry plane $(y=0$ and $y=b$ ) or, equivalently, $\partial u / \partial y=0$. This leads to

$$
\left\{\begin{array}{l}
\frac{\partial^{2} \psi_{\mathrm{I}}}{\partial y^{2}}(x, 0)=0, \quad 0 \leqslant x \leqslant a, \\
\frac{\partial^{2} \psi_{\mathrm{II}}}{\partial y^{2}}(x, 0)=\frac{\partial^{2} \psi_{\mathrm{II}}}{\partial y^{2}}(x, b)=0, \quad a \leqslant x .
\end{array}\right.
$$

On the solid boundary, the no-slip condition yields

$$
\begin{cases}\frac{\partial \psi_{\mathrm{I}}}{\partial y}(x, 1)=0, & 0 \leqslant x \leqslant a, \\ \frac{\partial \psi_{\mathrm{II}}}{\partial x}(a, y)=0, & 1 \leqslant y \leqslant b .\end{cases}
$$

The continuity conditions at $x=a, 0 \leqslant y \leqslant 1$, are

$$
\left\{\begin{array}{l}
\psi_{\mathrm{I}}(a, y)=\psi_{\mathrm{II}}(a, y), \\
\frac{\partial \psi_{\mathrm{I}}}{\partial x}(a, y)=\frac{\partial \psi_{\mathrm{II}}}{\partial x}(a, y), \\
\frac{\partial^{2} \psi_{\mathrm{I}}}{\partial^{2} x}(a, y)=\frac{\partial^{2} \psi_{\mathrm{II}}}{\partial x^{2}}(a, y), \\
\frac{\partial^{3} \psi_{\mathrm{I}}}{\partial^{3} x}(a, y)=\frac{\partial^{3} \psi_{\mathrm{II}}}{\partial x^{3}}(a, y) .
\end{array}\right.
$$

The aforementioned boundary conditions are specified based on the assumption that the stream function of the flow 
is symmetric with respect to the $x=0$ and periodic in the $y$ direction. In real applications, there may exist flow irregularities (or instabilities) with length scales larger than the characteristic lengths of the screen pump that break the flow symmetry. With these considerations, the flow solution may be valid in the region near the screen pump and deviate from the analytical solution because of the growth of the flow irregularities.

The homogeneous solution of the stream function $\psi_{h}$ satisfies the homogeneous biharmonic equation [16]

$$
\nabla^{4} \psi_{h}=0
$$

whose general solution can be expressed as eigensolution series. They are

$$
\begin{aligned}
\psi_{h \mathrm{I}}= & \sum_{n=1}^{\infty}\left[A_{n}^{f} Q_{n}^{f}(x)+B_{n}^{f} R_{n}^{f}(x)\right] \sin \left(\alpha_{n}^{f} y\right) \\
& +\sum_{n=1}^{\infty} C_{n}^{f} S_{n}^{f}(y) \cos \left(\beta_{n}^{f} x\right) \\
& +C_{0}^{f}\left(3 y-y^{3}\right)+\frac{q}{2}\left(3 y-y^{3}\right),
\end{aligned}
$$

for Region I and

$$
\begin{aligned}
\psi_{h \mathrm{II}}= & \sum_{n=1}^{\infty}\left[D_{n}^{f}+E_{n}^{f}(x-a)\right] \\
& \times e^{-\gamma_{n}^{f}(x-a)} \sin \left(\gamma_{n}^{f} y\right)+\frac{q y}{b}
\end{aligned}
$$

for Region II. The functions $Q_{n}^{f}, R_{n}^{f}$, and $S_{n}^{f}$ in Eqs. (26) and (27) are defined as

$$
\begin{aligned}
Q_{n}^{f}(x)= & e^{\alpha_{n}^{f}(x-a)}+e^{-\alpha_{n}^{f}(x+a)}, \\
R_{n}^{f}(x)= & x\left(e^{\alpha_{n}^{f}(x-a)}-e^{-\alpha_{n}^{f}(x+a)}\right), \\
S_{n}^{f}(y)= & e^{\beta_{n}^{f}(y-1)}-e^{-\beta_{n}^{f}(y+1)} \\
& -\left(\frac{1-e^{-2 \beta_{n}^{f}}}{1+e^{-2 \beta_{n}^{f}}}\right) y\left(e^{\beta_{n}^{f}(y-1)}+e^{-\beta_{n}^{f}(y+1)}\right)
\end{aligned}
$$

with $\alpha_{n}^{f}=n \pi, \beta_{n}^{f}=n \pi / a$, and $\gamma_{n}^{f}=n \pi / b$. Coefficients $A_{n}^{f}, B_{n}^{f}, C_{0}^{f}, C_{n}^{f}, D_{n}^{f}$, and $E_{n}^{f}$ are constants. These general solutions with the aforementioned particular solutions, Eqs. (A1) and (18), automatically fulfill the boundary conditions: Eq. (20), (21) 1 , and (22). With the remaining boundary conditions, Eqs. (21) 2 and (23), and the continuity conditions, Eq. (24), the system is completed for the unknown coefficients at a specified $q$.

Now, we need to specify the last condition for $q$. For the present microfluidic channel, the flow rate can be a function of the applied electric potential and the surface zeta potential as well as the applied pressure. In order to focus solely on the EO mechanism in the present paper, we assume there is no external pressure to drive the fluid. This condition states that the pressure at $x= \pm \infty$ is a constant, and, without the loss of generality, we set them to zero, i.e., $\left.p\right|_{x=-\infty}=\left.p\right|_{\infty}=0$. Furthermore, from the symmetry condition of the EO force, $K^{2} \phi_{e} \vec{\nabla} \phi_{p}$, and the velocity field, the pressure gradient [Eq. (6)] is a symmetric field with respect to $x=0$. This leads to that the pressure is an antisymmetric field with respect to $x=0$, and, hence, we have $p=0$ at $x=0$.

Before proceeding further, we comment on the benefit of using the present eigenseries solution instead of other numerical field solvers, such as finite element or volume methods, etc. The reason is that applying these field solver methods to the present EO flow may not be a straightforward procedure. Because for numerical convergence the boundary conditions in these field solvers are usually specified by the volume flow rate or the flow velocity at the channel inlet and by the constant pressure at the channel exit, the solved flows in general contain a driving pressure along the channel in addition to the electrokinetic force. To resolve the zero pressure at $x= \pm \infty$, an interactive procedure has to be applied. In addition, for large Debye-Hückel parameters, the EDL becomes thin, and this thin layer leads to a sharp transition of the flow velocity profile near the surface of the screen pump. The flow velocity varies exponentially in this layer, and the field solvers may loose accuracy if the computational mesh cannot provide a good resolution for the flow field.

The pressure in the two regions are found by integrating Eq. (6):

$$
\begin{aligned}
p_{\mathrm{I}}(x, y)= & 2 \sum_{n=1}^{\infty} B_{n}^{f} \alpha_{n}^{f}\left(e^{\alpha_{n}^{f}(x-a)}-e^{-\alpha_{n}^{f}(x+a)}\right) \\
& \times \cos \left(\alpha_{n}^{f} y\right)-2 \sum_{n=1}^{\infty} C_{n}^{f} \beta_{n}^{f}\left(\frac{1-e^{-2 \beta_{n}^{f}}}{1+e^{-2 \beta_{n}^{f}}}\right) \\
& \times\left(e^{\beta_{n}^{f}(y-1)}+e^{-\beta_{n}^{f}(y+1)}\right) \sin \left(\beta_{n}^{f} x\right)-3 x\left(q+2 C_{0}^{f}\right) \\
& +\int_{0}^{x} \nabla^{2}\left(\frac{\partial \psi_{p \mathrm{I}}}{\partial y}\right) d x-\int_{0}^{x} K^{2} \phi_{e \mathrm{I}} \frac{\partial \phi_{p \mathrm{I}}}{\partial x} d x
\end{aligned}
$$

and

$$
\begin{aligned}
p_{\mathrm{II}}(x, y)= & 2 \sum_{n=1}^{\infty} E_{n}^{f} \gamma_{n}^{f} e^{-\gamma_{n}^{f}(x-a)} \cos \left(\gamma_{n}^{f} y\right) \\
& +\int_{\infty}^{x} \nabla^{2}\left(\frac{\partial \psi_{p \mathrm{II}}}{\partial y}\right) d x-\int_{\infty}^{x} K^{2} \phi_{e \mathrm{II}} \frac{\partial \phi_{p \mathrm{II}}}{\partial x} d x .
\end{aligned}
$$

In Eqs. (29) and (30), the first integral terms are contribution from the particular solutions of the stream function; the second integral terms are from the electrokinetic forces, and the rest of the terms arise from the homogeneous stream function. The pressure is continuous across $x=a$. To simplify the calculation, we integrate the pressure from $y=0$ to 1 on both sides of $x=a$ and equate the resultant net forces. This leads to an additional equation for the volume flux $q$ for the pure EO driven flow:

$$
\begin{aligned}
& 2 \sum_{n=1}^{\infty} E_{n}^{f} \sin \gamma_{n}^{f}+3 a\left(q+2 C_{0}^{f}\right) \\
& \quad+\int_{0}^{1} \int_{\infty}^{a} \nabla^{2}\left(\frac{\partial \psi_{p \mathrm{II}}}{\partial y}\right) d x d y-\int_{0}^{1} \int_{0}^{a} \nabla^{2}\left(\frac{\partial \psi_{p \mathrm{I}}}{\partial y}\right) d x d y
\end{aligned}
$$




$$
\begin{aligned}
& +\int_{0}^{1} \int_{0}^{a} K^{2} \phi_{e \mathrm{I}} \frac{\partial \phi_{p \mathrm{I}}}{\partial x} d x d y \\
& -\int_{0}^{1} \int_{\infty}^{a} K^{2} \phi_{e \mathrm{II}} \frac{\partial \phi_{p \mathrm{II}}}{\partial x} d x d y=0 .
\end{aligned}
$$

To summarize, the equation for the volume flux Eq. (31), with the boundary conditions on the filter surface [Eqs. (21) and (23)] and the continuity conditions on the joint plane [Eq. (24)] complete the system for the unknowns $A_{n}^{f}, B_{n}^{f}, C_{0}^{f}$, $C_{n}^{f}, D_{n}^{f}, E_{n}^{f}$, and $q$. For numerical calculation, we discretize the boundaries into uniformly distributed computational collocation points, substitute the coordinates into the equation system and truncate the infinite series in the solutions into a sufficiently large but finite system, and form a system of linear algebraic equations for the aforementioned unknowns. In the next section, we will first demonstrate the numerical convergence and verify the asymptote of the solution to a simple straight channel, for which the exact solution is known. Then the flow with a range of the Debye-Hückel parameters and various geometric configurations are investigated.

\section{RESULTS AND DISCUSSION}

\section{A. Convergence}

First, let us compare our model with the analytic solution of the flow passing through an infinite microchannel with a constant width. With this simple geometry, the volume flux $q$ can be solved straightforwardly following the solving procedures outlined in Sec. II, which reads

$$
q=1-\frac{\sinh (K y)}{K \cosh (K)} .
$$

We inspect the asymptotic behavior of two sets of examples. The first set is to verify the truncation errors with the various summation terms $N$ at a fixed Debye-Hückel parameter $K$. We choose $K=10$ and calculate the solutions with the channel length $a$ from 10 to 100 . The number of summation terms are $N=20,40$, and 100 . The channel width $b$ is set to 1 , i.e., the pump is a slit array composed of infinitesimally thin plates. The convergence is shown in Table I. It is obvious that the numerical results asymptote to the exact solution with increasing as well as with the increasing length of the filter channel $a$.

The other cases are simulated with a fixed $N=100$ but with a set of distinct values of the Debye-Hückel parameters: $K=0.5, K=2$, and $K=10$, and the pump length $a$ from 10

TABLE I. The volume flux with various channel lengths $a$ and various number of summation terms. The channel width $b$ is set to 1 , and the Debye-Hückel parameter is $K=10$. The results asymptote to the exact solution of the infinite long microchannel.

\begin{tabular}{lcccc}
\hline \hline$a$ & $N=20$ & $N=40$ & $N=100$ & Exact \\
\hline 10 & 0.897 & 0.896 & 0.895 & 0.900 \\
20 & 0.900 & 0.899 & 0.898 & 0.900 \\
40 & 0.900 & 0.900 & 0.899 & 0.900 \\
80 & 0.900 & 0.900 & 0.900 & 0.900 \\
100 & 0.900 & 0.900 & 0.900 & 0.900 \\
\hline \hline
\end{tabular}
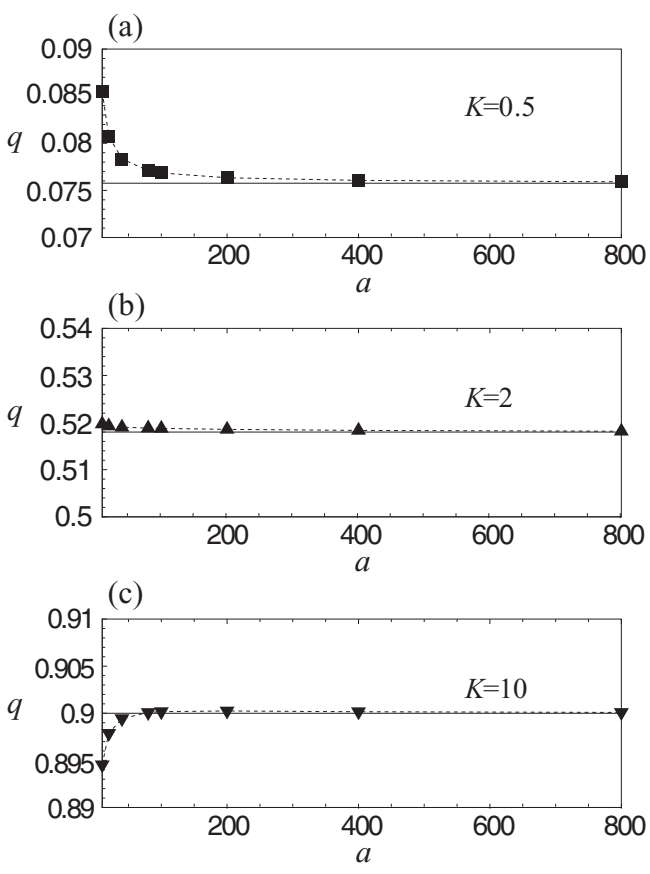

FIG. 4. The asymptotic behavior (dashed lines with distinct symbols) approaching to the exact flux (solid lines) of infinitely long (continuous) channels for (a) $K=0.5$, (b) $K=2$, and (c) $K=10$. The results of the screen pump approach to the theoretical values of the continuous channels when the channel length $a$ increases.

to 100 . The simulated results of the volume flux together with the exact solution are shown in Fig. 4. The flat horizontal solid lines represent the volume flux of the flow in the infinite channel, and the dashed lines with symbols denote the dependence of the volume fluxes on the pump length as predicted by the present theory. It is interesting to notice that the asymptotic behaviors can be divided in two ways. For the cases with the smaller $K(0.5$ and 2$)$, the volume fluxes approach those of the infinite channel from the side with larger flux, but for $K=10$, the volume flux asymptotes to the long channel solution from the lower flux side.

The reason causing the alternation of the asymptotic behaviors can be explained by the alternation of the net effective driving force as the channel length varies. The electrokinetic force is a body force produced by the interaction between the charge density in the fluid and the applied electric field, and the fluid viscous force is produced by the fluid strain rate generated by the flow field. Because the thickness of the charged layer is reversely proportional to the Debye-Hückel parameter $K$, the electric charge is distributed farther into the fluid column, as well as the nonvanishing strain rate field when $K$ becomes smaller. This provides extra driving capability from the regions near the entrance and exit of the slit array for small channel length $a$, and, hence, the volume flux increases. On the other hand, when $K$ is large, the charge remains confined in a thin layer against the solid boundary as well as the strain field. As $a$ becomes small, the driving force, restricted in the thin region adjacent to the boundary, also becomes proportionally small and can no longer effectively propel the fluid column as the long channels can and, hence, reduces the volume flux. 
TABLE II. The volume flux with various channel lengths $(a, b)$ and various number of summation terms. The Debye-Hückel parameter is $K=0.1$.

\begin{tabular}{|c|c|c|c|c|c|}
\hline$(a, b)$ & $N=20$ & $N=40$ & $N=60$ & $N=80$ & $N=100$ \\
\hline$(0.1,1.05)$ & 0.0613 & 0.0614 & 0.0616 & 0.0617 & 0.0617 \\
\hline$(1.0,1.15)$ & 0.0238 & 0.0238 & 0.0239 & 0.0239 & 0.0239 \\
\hline$(5.0,1.30)$ & 0.0097 & 0.0097 & 0.0097 & 0.0097 & 0.0097 \\
\hline
\end{tabular}

\section{B. The volume flux versus different geometries and Debye-Hückel parameter}

We now investigate the geometric effect of the screen pump and the Debye-Hückel parameter $K$ on the flow. Six distinct values of $K$ are selected, and, for each value, the volume fluxes of a range of channel lengths and widths are calculated. Because of the discretization and truncation of the series, Gibbs oscillations exist on the joining plane $(x=a)$ between Regions I and II. Larger offset of the Gibbs oscillations is found near the corner of the screen pump block, $x=a, y=1$, which is a singular point of the flow field. Similar phenomena are described in Ref. [17] for Stokes flows in cavities. The convergence versus the number of terms of the eigensolutions for a set of filter parameters is summarized in Table II. From as few as 20 terms, the resultant series produce errors within a thousandth. The fast convergence has been seen in similar applications [16,18].

The trends of the fluxes with distinct pump geometries are shown in Fig. 5. In each panel, the horizontal axis is the free-flow field width $b$; the vertical axis is the volume flux, and the lines represent the volume fluxes for three different channel lengths $a$. Drawing our attention to the volume fluxes at $b=1$, we immediately see that the volume fluxes increase as $a$ decreases for small $K(K=0.1,1.0)$, and the trend reverses for $K=10$. The alternation takes place at about $K=2$. This agrees with the findings in the previous section. The surface areas of the screen pump at $x= \pm a$ are perpendicular to the direction of the volume flow rate, and they are controlled by parameter $b$. These areas play two different roles. When $K$ is small, the electrokinetic force is weak, the surface area blocks the flow, and the volume fluxes decrease. On the other hand, when $K$ is large $(K=1,10)$, the electrokinetic force increases with increasing $b$, and this proportionality leads to an increase in the volume fluxes as $b$ is increased in the computed domain $(1 \leqslant b \leqslant 1.4)$.

The flow fields at $K=1,10,100$, and 200 with geometric parameters $a=4$ and $b=1.5$ are plotted in Fig. 6. They are presented by the contour lines, streamlines, of the stream function whose values are equally incremental. The streamlines are in parallel to the solid or slip boundaries apart from the joint area, near $x=a$, where the flows expand smoothly around the corner. For small $K$ [Fig. 6(a)] a thick shear layer is found near the block surface. For larger $K$, e.g., comparing $K=100$ and 200 with the same set of streamline values, the streamlines are more evenly distributed, indicating that the flow velocity becomes uniform across the channel width direction. In addition, the streamlines around the corner are shifted further toward the boundary because the electrokinetic force is confined closer to the solid surface. We (a)

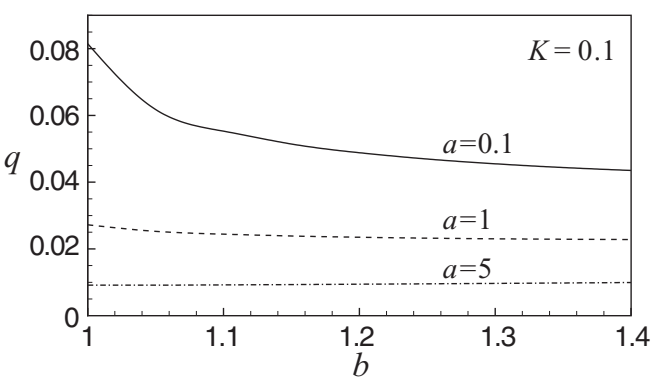

(b)

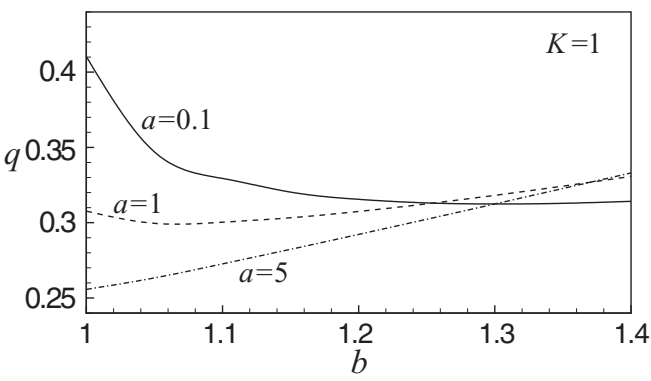

(c)

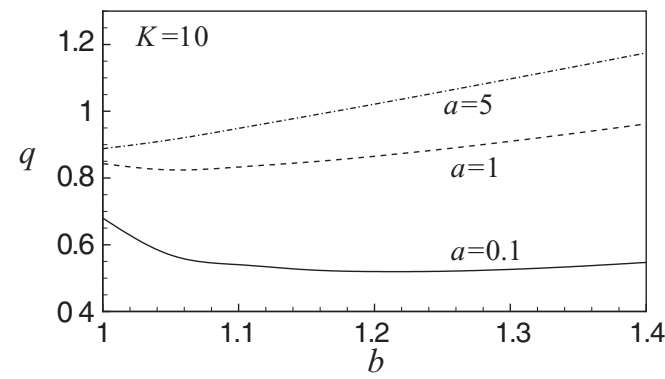

FIG. 5. The volume flux versus the geometry of the screen pump and the Debye-Hückel parameter $K$. From top, (a) $K=0.1$, (b) $K=1$, and (c) $K=10$. The horizontal axes are the free-flow field width $b$. Three channel lengths $a=0.1,1$, and 5 are depicted by lines in each panel.

must notice that, for fulfilling the no-slip boundary condition and the uniform flow in the channel, there exists a thin shear layer against the solid surface and the electrokinetic force concentrates within the layer. We refer to this layer as the boundary layer although it is rooted on a different mechanism from the classical boundary layer theory. This leads to an interesting finding that is described in the next section.

\section{Generation of recirculating eddies}

For high values of $K$, the boundary layer becomes very thin, and the electrokinetic force is confined in the thin layer against the filter surface. As the result, the fluid is pulled by the layer and then driven by the diffusive fluid viscosity. The interplay between the two effects lead to an unexpected alternation of the flow direction.

This phenomenon is found in a thin screen pump whose geometric parameters are $a=0.1, a=0.01$, and $b=1.5$. The flow fields of three values of $K$ are sketched in Figs. 7 and 8 . In $K=1$, the flow is driven smoothly around the filter corner and expands into the parallel free stream away from the filter. On the other hand, a recirculation eddy is formed 


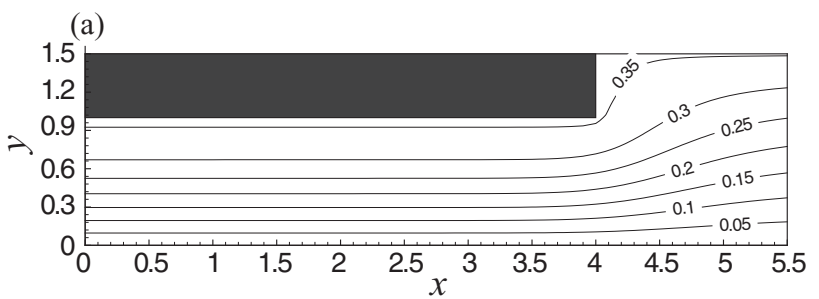

(c)

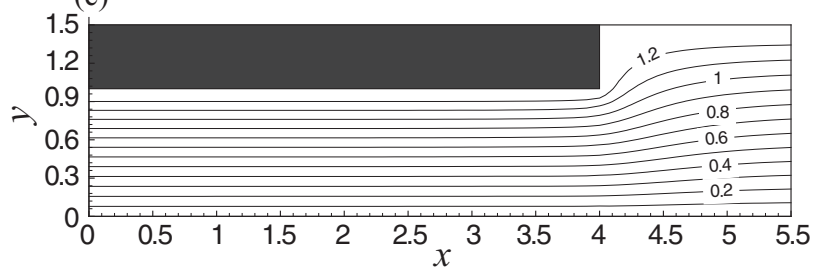

(b)

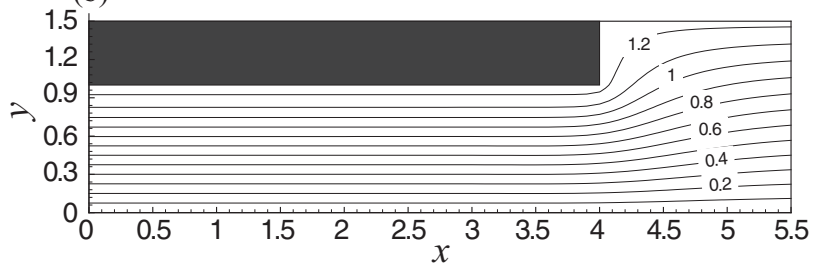

(d)

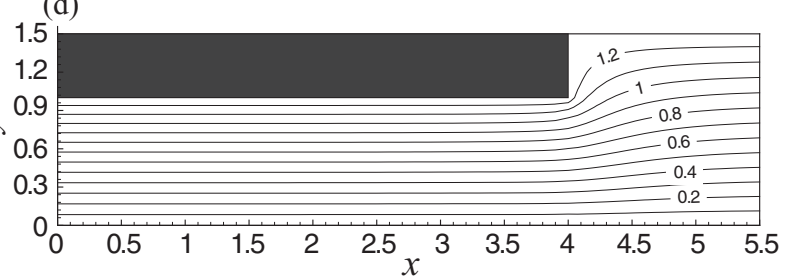

FIG. 6. The flow fields with the screen pump geometry $a=4$ and $b=1.5$. Four values of $K$ are plotted: (a) $K=1$, (b) $K=10$, (c) $K=100$, and (d) $K=200$.

when $K$ is larger. This is because the electrokinetic force is constrained near the surface but the viscosity is not strong enough in bringing the fluid to overcome the static pressure far downstream. The eddy occupies the central of the flow channel and results in a dramatic drop in the transported volume flux. The effective flow channel reduces to the layer along the field boundary. As $K$ increases, the eddy is further attracted toward the filter and completely blocks the flow channel, which provides the positive volume flux. Interestingly, a new central flow stream with a reversed flow direction is created by the eddy.

\section{CONCLUDING REMARKS}

In this study we proposed a screen pump for electro-osmotic flow and have successfully estimated the flux driven by the (a)

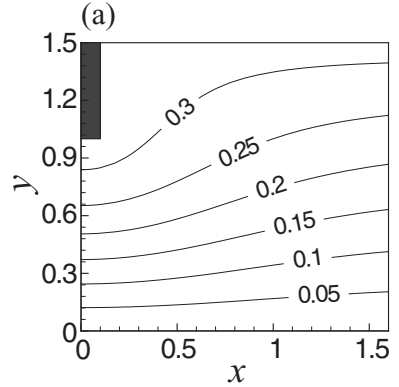

(c)

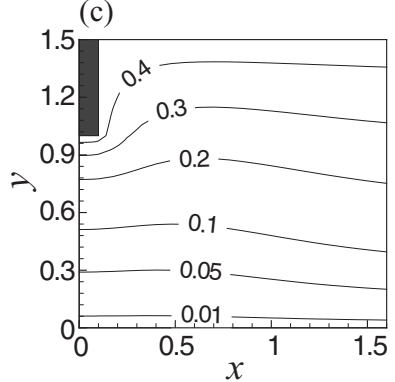

(b)

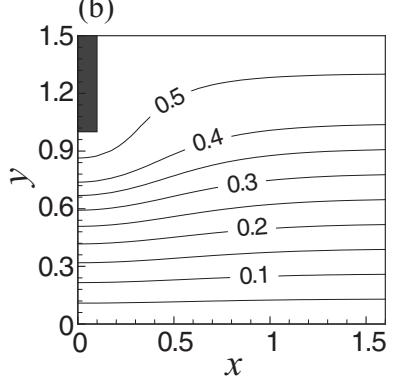

(d)

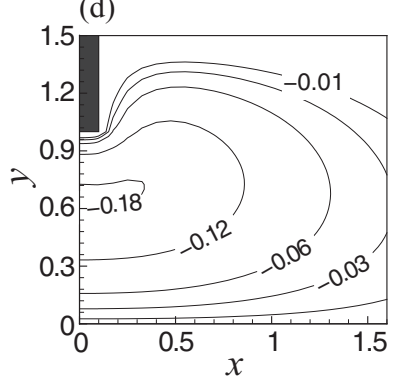

FIG. 7. The flow fields with the screen pump geometry $a=0.1$ and $b=1.5$. Four values of $K$ are plotted: (a) $K=1$, (b) $K=10$, (c) $K=100$, and (d) $K=200$. pump without an external driving pressure. Under the DebyeHückel approximation, the analytic solutions for the applied potential, EDL field, flow stream function, and pumping rate were obtained in the form of series expansions.

By letting $a \rightarrow \infty$, the analytic solutions converge to the exact solution of the electro-osmotic flow in an infinitely long microchannel (continuous channel). The approaches the exact solution of the continuous channel are different with different values of the Debye-Hückel parameter $K$. For small $K$, because of the thick EDL, the volume flux approaches decreasingly the exact solution of the continuous channel with increasing the channel length $a$. On the other hand, for large $K$, the volume flux approaches increasingly the exact solution of the continuous channel.
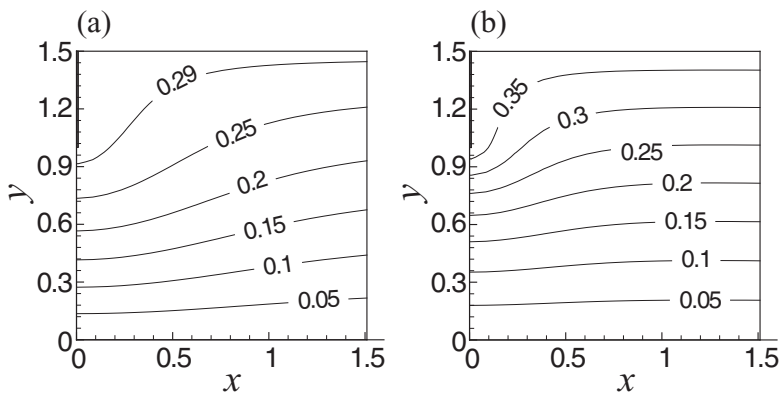

(c)

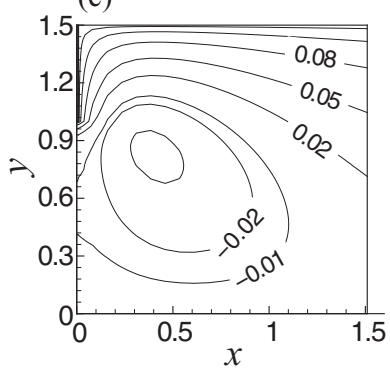

(d)

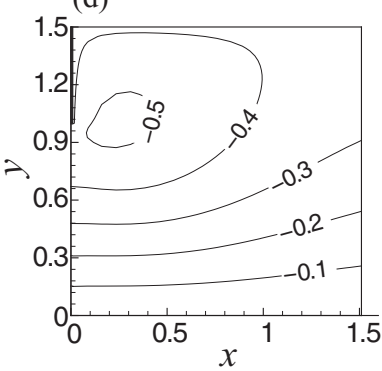

FIG. 8. The flow fields with the screen pump geometry $a=0.01$ and $b=1.5$. Four values of $K$ are plotted: (a) $K=1$, (b) $K=10$, (c) $K=100$, and (d) $K=200$. Note that in this thin screen pump, recirculating eddies appear, and they lead to reversing flow at large $K, K=200$, for example. 
It is obvious that the electro-osmotic flow rate of the screen pump is a function of the geometric parameters $a, b$ and the Debye-Hückel parameter $K$. In general, when $a \geqslant 1$ the electrokinetic volume flow rate increases with increasing $b$. This trend is because the total electric-kinetic driving force increases as these parameters increase. However, there are more delicate influences by these parameters on the flow field. It happens when $a$ is small $(a<1)$ : The screen pump blocks the flow because the viscous drag force outperforms the electrokinetic driving force, and the total resultant force becomes reversely dependent on $b$.

One intriguing reverse flow is also found when the ratio $a / b$ is small and the Debyue-Hückel parameter $K$ is large. This screen pump is composed of thin vertically aligned screen blocks. The thin EDL, large $K$, indicates the electrokinetic force propels the flow in the thin boundary layer against the block surface, and the diffusive viscous force induces recirculating eddies attached to the screen block. Then these recirculating eddies induce a reverse flow. It is therefore possible to design the direction of the flow in microfluids using this screen pump device.

\section{ACKNOWLEDGMENTS}

The work is supported in part by the National Taiwan University and by the National Research Council of Taiwan under Contract Nos. NSC 99-2628-M-002-003 and NSC 98-2811-E-002-112.

\section{APPENDIX A: THE PARTICULAR SOLUTION IN REGION I}

Here we display the solution form and corresponding coefficients we chose for the particular solution in Region I, that is,

$$
\begin{aligned}
& \psi_{p \mathrm{I}}(x, y)=\sum_{n=1}^{\infty} Q_{n}^{1}\left[\sin \left(\beta_{n}^{e} y\right)+A_{n}^{1} y+B_{n}^{1} y^{3}\right]+\sum_{n=1}^{\infty} Q_{n}^{2}\left[\sin \left(\beta_{n}^{e} y\right)+A_{n}^{2} \sin \left(\alpha_{n}^{e} y\right)+B_{n}^{2} y \cos \left(\alpha_{n}^{e} y\right)\right] \\
& +\sum_{m, n=1}^{\infty} Q_{m n}^{3}\left\{\sin \left[\left(\beta_{n}^{e}-\alpha_{m}^{p}\right) y\right]+A_{m n}^{3} \sin \left(\alpha_{m}^{p} y\right)+B_{m n}^{3} y \cos \left(\alpha_{m}^{p} y\right)\right\} \\
& +\sum_{m, n=1}^{\infty} Q_{m n}^{4}\left\{\sin \left[\left(\beta_{n}^{e}+\alpha_{m}^{p}\right) y\right]+A_{m n}^{4} \sin \left(\alpha_{m}^{p} y\right)+B_{m n}^{4} y \cos \left(\alpha_{m}^{p} y\right)\right\} \\
& +\sum_{m, n=1}^{\infty} Q_{m n}^{5}\left\{\sin \left[\left(\beta_{n}^{e}-\alpha_{m}^{p}\right) y\right]+A_{m n}^{5} \sin \left[\left(\alpha_{n}^{e}+\alpha_{m}^{p}\right) y\right]+B_{m n}^{5} y \cos \left[\left(\alpha_{n}^{e}+\alpha_{m}^{p}\right) y\right]\right\} \\
& +\sum_{m, n=1}^{\infty} Q_{m n}^{6}\left\{\sin \left[\left(\beta_{n}^{e}-\alpha_{m}^{p}\right) y\right]+A_{m n}^{6} \sin \left[\left(\alpha_{n}^{e}-\alpha_{m}^{p}\right) y\right]+B_{m n}^{6} y \cos \left[\left(\alpha_{n}^{e}-\alpha_{m}^{p}\right) y\right]\right\} \\
& +\sum_{m, n=1}^{\infty} Q_{m n}^{7}\left\{\sin \left[\left(\beta_{n}^{e}+\alpha_{m}^{p}\right) y\right]+A_{m n}^{7} \sin \left[\left(\alpha_{n}^{e}+\alpha_{m}^{p}\right) y\right]+B_{m n}^{7} y \cos \left[\left(\alpha_{n}^{e}+\alpha_{m}^{p}\right) y\right]\right\} \\
& +\sum_{m, n=1}^{\infty} Q_{m n}^{8}\left\{\sin \left[\left(\beta_{n}^{e}+\alpha_{m}^{p}\right) y\right]+A_{m n}^{8} \sin \left[\left(\alpha_{n}^{e}-\alpha_{m}^{p}\right) y\right]+B_{m n}^{8} y \cos \left[\left(\alpha_{n}^{e}-\alpha_{m}^{p}\right) y\right]\right\},
\end{aligned}
$$

where the coefficients are known functions in terms of the coefficients and parameters of the electric and EDL potentials:

$$
\begin{gathered}
Q_{n}^{1}=-\frac{K^{4} a_{n} A_{0}^{p}}{\left(\beta_{n}^{e}\right)^{3}\left(\left(\beta_{n}^{e}\right)^{2}+k^{2}\right)}, \\
Q_{n}^{2}=-\cosh \left(\alpha_{n}^{e} x\right) \frac{K^{2} \beta_{n}^{e} A_{n}^{e} A_{0}^{p}}{\left[\left(\alpha_{n}^{e}\right)^{2}-\left(\beta_{n}^{e}\right)^{2}\right]^{2}}, \\
Q_{m n}^{3}=\cosh \left(\alpha_{m}^{p} x\right) \\
\times \frac{K^{4} \beta_{n}^{e} \alpha_{m}^{p} a_{n} A_{m}^{p}}{2\left[\left(\beta_{n}^{e}\right)^{2}+K^{2}\right]\left[\left(\alpha_{m}^{p}\right)^{2}-\left(\beta_{n}^{e}-\alpha_{m}^{p}\right)^{2}\right]^{2}},
\end{gathered}
$$

$$
\begin{aligned}
Q_{m n}^{4}= & \cosh \left(\alpha_{m}^{p} x\right) \\
& \times \frac{K^{4} \beta_{n}^{e} \alpha_{m}^{p} a_{n} A_{m}^{p}}{2\left[\left(\beta_{n}^{e}\right)^{2}+K^{2}\right]\left[\left(\alpha_{m}^{p}\right)^{2}-\left(\beta_{n}^{e}+\alpha_{m}^{p}\right)^{2}\right]^{2}}, \\
Q_{m n}^{5}= & \cosh \left[\left(\alpha_{n}^{e}+\alpha_{m}^{p}\right) x\right] \\
& \times \frac{K^{2}\left(\beta_{n}^{e}+\alpha_{n}^{e}\right) \alpha_{m}^{p} A_{n}^{e} A_{m}^{p}}{4\left[\left(\alpha_{n}^{e}+\alpha_{m}^{p}\right)^{2}-\left(\beta_{n}^{e}-\alpha_{m}^{p}\right)^{2}\right]^{2}}, \\
Q_{m n}^{6}= & \cosh \left[\left(\alpha_{n}^{e}-\alpha_{m}^{p}\right) x\right] \\
& \times \frac{K^{2}\left(\beta_{n}^{e}-\alpha_{n}^{e}\right) \alpha_{m}^{p} A_{n}^{e} A_{m}^{p}}{4\left[\left(\alpha_{n}^{e}-\alpha_{m}^{p}\right)^{2}-\left(\beta_{n}^{e}-\alpha_{m}^{p}\right)^{2}\right]^{2}},
\end{aligned}
$$




$$
\begin{aligned}
& Q_{m n}^{7}= \cosh \left[\left(\alpha_{n}^{e}+\alpha_{m}^{p}\right) x\right] \\
& \times \frac{K^{2}\left(\beta_{n}^{e}-\alpha_{n}^{e}\right) \alpha_{m}^{p} A_{n}^{e} A_{m}^{p}}{4\left[\left(\alpha_{n}^{e}+\alpha_{m}^{p}\right)^{2}-\left(\beta_{n}^{e}+\alpha_{m}^{p}\right)^{2}\right]^{2}}, \\
& Q_{m n}^{8}= \cosh \left[\left(\alpha_{n}^{e}-\alpha_{m}^{p}\right) x\right] \\
& \times \frac{K^{2}\left(\beta_{n}^{e}+\alpha_{n}^{e}\right) \alpha_{m}^{p} A_{n}^{e} A_{m}^{p}}{4\left[\left(\alpha_{n}^{e}-\alpha_{m}^{p}\right)^{2}-\left(\beta_{n}^{e}+\alpha_{m}^{p}\right)^{2}\right]^{2}}, \\
& A_{n}^{1}=\frac{3(-1)^{n}}{2}, \quad B_{n}^{1}=\frac{(-1)^{n}}{2}, \\
& A_{n}^{2}= F\left(\alpha_{n}^{e}, \beta_{n}^{e}\right), \quad B_{n}^{2}=G\left(\alpha_{n}^{e}, \beta_{n}^{e}\right), \\
& A_{m n}^{3}=F\left(\alpha_{m}^{p}, \beta_{n}^{e}-\alpha_{m}^{p}\right) \\
& B_{m n}^{3}=G\left(\alpha_{m}^{p}, \beta_{n}^{e}-\alpha_{m}^{p}\right), \\
& A_{m n}^{4}=F\left(\alpha_{m}^{p}, \beta_{n}^{e}+\alpha_{m}^{p}\right), \\
& B_{m n}^{4}=G\left(\alpha_{m}^{p}, \beta_{n}^{e}+\alpha_{m}^{p}\right),
\end{aligned}
$$

$$
\begin{aligned}
& A_{m n}^{5}=F\left(\alpha_{n}^{e}+\alpha_{m}^{p}, \beta_{n}^{e}-\alpha_{m}^{p}\right), \\
& B_{m n}^{5}=G\left(\alpha_{n}^{e}+\alpha_{m}^{p}, \beta_{n}^{e}-\alpha_{m}^{p}\right), \\
& A_{m n}^{6}=F\left(\alpha_{n}^{e}-\alpha_{m}^{p}, \beta_{n}^{e}-\alpha_{m}^{p}\right), \\
& B_{m n}^{6}=G\left(\alpha_{n}^{e}-\alpha_{m}^{p}, \beta_{n}^{e}-\alpha_{m}^{p}\right), \\
& A_{m n}^{7}=F\left(\alpha_{n}^{e}+\alpha_{m}^{p}, \beta_{n}^{e}+\alpha_{m}^{p}\right), \\
& B_{m n}^{7}=G\left(\alpha_{n}^{e}+\alpha_{m}^{p}, \beta_{n}^{e}+\alpha_{m}^{p}\right), \\
& A_{m n}^{8}=F\left(\alpha_{n}^{e}-\alpha_{m}^{p}, \beta_{n}^{e}+\alpha_{m}^{p}\right), \\
& B_{m n}^{8}=G\left(\alpha_{n}^{e}-\alpha_{m}^{p}, \beta_{n}^{e}+\alpha_{m}^{p}\right) .
\end{aligned}
$$

The auxiliary functions $F$ and $G$ are

$$
\begin{gathered}
F(\alpha, \beta)=\frac{-\cos \alpha \sin \beta+\alpha \sin \alpha \sin \beta+\beta \cos \alpha \cos \beta}{\sin \alpha \cos \alpha-\alpha}, \\
G(\alpha, \beta)=\frac{\alpha \cos \alpha \sin \beta-\beta \sin \alpha \cos \beta}{\sin \alpha \cos \alpha-\alpha} .
\end{gathered}
$$

[1] J. P. Urbanski, T. Thorsen, J. A. Levitan, and M. Z. Bazant, Appl. Phys. Lett. 89, 143508 (2006).

[2] H. Zhao and H. H. Bau, Phys. Rev. E 75, 066217 (2007).

[3] N. G. Green, A. Ramos, A. Gonzalez, H. Morgan, and A. Castellanos, Phys. Rev. E 61, 4011 (2000).

[4] K. A. Rose, B. Hoffman, D. Saintillan, E. S. G. Shaqfeh, and J. G. Santiago, Phys. Rev. E 79, 011402 (2009).

[5] C. Marquet, A. Buguin, L. Talini, and P. Silberzan, Phys. Rev. Lett. 88, 168301 (2002).

[6] R. Sadr, M. Yoda, Z. Zheng, and A. T. Conlisk, J. Fluid Mech. 506, 357 (2004).

[7] S. Bhattacharyya, Z. Zheng, and A. T. Conlisk, J. Fluid Mech. 540, 247 (2005).
[8] B. Zaltzman and I. Rubinstein, J. Fluid Mech. 579, 173 (2007).

[9] S. Ghosal, J. Fluid Mech. 459, 103 (2002).

[10] T. L. Sounart and J. C. Baygents, J. Fluid Mech. 576, 139 (2007).

[11] H. Bruus, Theoretical Microfluidics (Oxford, New York, 2008).

[12] T. M. Squires and S. R. Quake, Rev. Mod. Phys. 77, 977 (2005).

[13] C. Y. Kuo, C. Y. Wang, and C. C. Chang, Electrophoresis 29, 4386 (2008).

[14] S. Nagrath et al., Nature (London) 450, 1235 (2007).

[15] D. A. Saville, Annu. Rev. Fluid Mech. 9, 321 (1977).

[16] C. Y. Wang, Phys. Fluids A 5, 1113 (1993).

[17] S. Qian and H. H. Bau, Appl. Math. Mod. 29, 726 (2005).

[18] C.-O. Ng and C. Y. Wang, Phys. Fluids 21, 013602 (2009). 